\title{
Cloud Computing Adoption in the Healthcare Sector: A SWOT
} Analysis

\author{
Maslin Masrom $^{1} \&$ Ailar Rahimli $^{1}$ \\ ${ }^{1}$ Razak School of Engineering and Advanced Technology, Universiti Teknologi Malaysia, Kuala Lumpur, \\ Malaysia \\ Correspondence: Ailar Rahimli, Razak School of Engineering and Advanced Technology, Universiti Teknologi \\ Malaysia, Jalan Semarak, 54100 Kuala Lumpur, Malaysia. Tel: 60-14-701-5384. E-mail: a.rahimly@gmail.com
}

$\begin{array}{lc}\text { Received: August 3, } 2014 & \text { Accepted: January 19, } 2015 \quad \text { Online Published: April 13, } 2015 \\ \text { doi:10.5539/ass.v11n10p12 } & \text { URL: http://dx.doi.org/10.5539/ass.v11n10p12 }\end{array}$

\begin{abstract}
Emergence of cloud computing bring new evolution in information technology (IT) industry. Cloud computing is growing interest to several organizations all over the world but adoption of cloud computing is not easy task because there are many barriers associated with adopting it, which should be eliminated. In the healthcare sector the rate of adopting the cloud computing is low and several barriers involve in adopting the cloud computing in the healthcare sector that major barrier is security. The aim of this paper is to investigate the cloud computing adoption in the healthcare sector using SWOT (Strength, Weaknesses, Opportunities and Threats) analysis. The results reveal the cloud computing adoption is one of the suitable solutions based on SWOT analysis for healthcare sector issues such as: cost, storage, collaborating.
\end{abstract}

Keywords: information technology, cloud computing adoption, healthcare sector, and SWOT analysis

\section{Introduction}

Cloud computing is one of the solution for several organizations because it has ability to develop the organizations. Cloud computing is suitable to organizations for managing data effectively and using a flexible, scalable, and affordable platform. With this solution many organizations can exchange data among different system without considering to the infrastructure controlling that data. So, various businesses such as large business, higher education, small and medium businesses over the world are finding that with cloud computing, they can develop and increase efficiency and productivity without increasing in the capital expenditure. However, the benefits of cloud computing are obvious for most of the industries but the healthcare sector has been followed it slowly because very hesitant for concerns about security and privacy. Healthcare needs suitable innovation to stay cost effectiveness, timely, and efficiency. Many IT experts believe cloud computing is best solution to improve healthcare sector. Cloud computing is defined as an on demand service that is self-services that user can access to the computational resources in anytime and anywhere (Bamiah et al., 2012). Cloud computing is new technology that deliver the service over the Internet without need to setting up of complex infrastructure. Cloud computing provide services such as extra storage, capacity, and advanced services are available for user and user should pay based on usage (Koufi et al., 2010). Therefore, the aim of this study is to investigate the cloud computing adoption as a best solution for healthcare sector based on SWOT analysis.

This paper is organized as follows. In the first part, describe the cloud computing completely that include: structure, types, segments, features, and advantages and disadvantages. In the second part discuss about the cloud computing in the healthcare sector, in the third part, present the SWOT analysis for cloud computing with view to healthcare sector, and last part, provide conclusion.

\section{Cloud Computing}

\subsection{What's Cloud Computing?}

Cloud computing is a defined as a technology that with cloud computing user do not need to own the resources like hardware, software, and user can use advance service from cloud computing over the internet in anytime and anywhere. Cloud computing has ability to provide computing resources and relive the various organizations from heavy investment for software, hardware, resource infrastructure, and platform. The main difference between the cloud computing and other compute paradigms is that the cloud computing has ability to offer infinite the 
capacity and power (Koufi et al., 2010).

Cloud computing components are as below (Chowdhary et al., 2011):

- Virtualization: partitioned and scalable infrastructure is provided for applications with virtualization. Virtual machine is created with API and web interface. The application determines how virtual components are to be configured and interconnected.

- Computation: cloud computing is developed the technology like, distributed computing, and grid computing.

- Connectivity: its spans the web.2 framework and application service level agreement (SLA).

- Architecture: the cloud computing architecture cover the service oriented architecture (SOA) that is implemented user application. Cloud computing provide services normally on form of web services.

- Services: over the cloud computing, user can use services such as: SaaS, IaaS, PaaS, and HaaS. Cloud interacts with user applications and services through APIs.

\subsection{Cloud Computing Structure}

The cloud computing structure is layer architecture. Each layer provide specific service and cloud computing has three main layers, first layer is SaaS, software as a service is an upper layer that let user to run applications from cloud. Second layer is PaaS, platform as a service provide operational system for implementing the applications from cloud. Last layer is IaaS, infrastructure as a service, this layer offers storage and computing resources as a services. The goal of this layers same as the PaaS but this layer is referred to hardware (Deng et al., 2011).

\subsection{Diffrent Type of Cloud Computing}

Cloud computing has four types that contain: public cloud, private, hybrid and community cloud. Any user and organization can use public cloud services from third party that may available free or cost can be considered. Private cloud is owned by organization and for private use. Hybrid cloud is combining two cloud infrastructures and it can provide extra resources and services for high demand. Community cloud is like a private cloud but in this cloud, resources are shared among the member of closed group who has same requirements (Deng et al., 2011).

\subsection{Segment of Cloud Computing}

Cloud computing has three main segments: application, storage and connectivity. The application segment is the part of the Internet technology that is already proved as the helpful and constructive model. The main concept of cloud computing is called storage or infrastructure. All cloud providers will allow users to create their own cloud application with limited space. Connectivity is most important part of cloud computing. High-speed Internet connectivity is very necessary for connecting to the cloud. Without the Internet connection cannot to use of storage and application of cloud (Chowdhary et al., 2011).

\subsection{Features of Cloud Computing}

Various features of cloud computing are as below (Moschella et al., 2004):

- Scalability and Elasticity: scalability and elasticity is core future and very essential for cloud computing. It's very important feature of this service that any development and modification in the service are very fast and easy that makes this service very resilient and scalable. One can easily add up needed processing speed, data storage and bandwidth or number of license in short time. One requirement not to plan any more for project implementation, project costing, project closer, and procurement; but one requirement to place a purchase order to service provider to get service in due time.

- Multi tenancy: multi tenancy is a highly fundamental issue in the cloud computing, where the location of data or code is mainly unknown and the same reason may be assigned to the several users. This effect infrastructure resource also application and data services that are hosted on shared resources but require to be made available in several isolated examples.

- Energy consumption: energy consumption is related to decrease the additional cost of energy consumption. Cloud is based on the network environment and so, mainly allows decreasing the energy consumption.

- Reliability: reliability is an amazing feature of cloud computing. Reliability is one of the essential cloud computing characteristic that will increase when redundant websites are accessed. Reliability is enhanced via having the several sites for the same service, such that if one confronts disconnections, the other can take over the load.

- Security: security is clearly essential in the all system that has sensitive data and code. The cloud is 
administered and managed by IT expert so the data will be secured in system crash and data loss (Deng et al., 2011).

- Consumption based billing: the capability to estimate the cost based on actual consumption of resource is a related feature of cloud computing. Pay per using, if user don't use the service and resource, they pay the nothing so it is one of the great feature of cloud computing.

- Data management: it's an essential aspect in sense of storage, where data has flexibility distributed among several resources. Data consistency and implicitly require to be maintained over the wide distribution of replicated data source. Concurrently, the system always requires being aware of the location of data at the time of replicating data among data centers. So, data management is a grant feature of cloud computing.

- Self-service model: one of the reason for popularity of cloud computing is self-service model. The service is an available for user based on demand. For example, user has ability to build programs, upload field and manage and generate reports.

\subsection{Advantages and Disadvantages of Cloud Computing}

Cloud computing such as the other new technology has both advantages and disadvantages that require considering before implementation (Deng et al., 2011; Ahuja et al., 2011; Chowdhary et al., 2011; Fern'ndez et al., 2012; Moschella et al., 2004).

Advantages

1) Cost effectiveness

2) Innovation and flexible

3) Easy access (accessibility)

4) Simplify cost and consumption

5) Enterprise Grade Service and Management

6) Faster provisioning of applications and systems

7) Ease of integration

8) Highly secure infrastructure

9) Compliant processes and facilitates

10) Resilient and flexible in disaster recovery

11) Reduce the cost of the hardware, software and maintenance

12) Access to infinite storage

Disadvantages

1) Data loss

2) Failure in compliance

3) Require to constant connectivity

4) Dependency

5) Quality problem that related to the cloud service provider

6) Time and budget limited

7) Privacy and security concerns

\section{Cloud Computing in Healthcare Sector}

According to the features of the cloud computing that mention in the previous part, there are suitable reasons to use the cloud computing in the healthcare sector. By using the cloud computing, many problems of the healthcare sector could be solved such as: maximizing storage capacity, reducing operating cost, and optimizing the resource (Chowdhary et al., 2011; Bamiah et al., 2012).

Due to growing in the number of the electronic file in the hospital system, this system face with several problems and move to the cloud is good idea (Bamiah et al., 2012; De la Torre-Díez et al., 2012; Al Masud \& Rashid, 2012). Moving the cloud offer the several benefits to the healthcare sector but also bring some risks that related to the security and privacy. The most important benefit is quality of service because with using the cloud computing in the healthcare sector, the collaboration among the doctors and patients will be increased and easier; 
lead to increase quality of service of patients. The healthcare system include huge amount of patient data that are growing in its volume, variety and veracity that create two main problems for the healthcare system that include complexity and increase the need of the IT experts. The maintaining and managing of these data are very essential so need to software, hardware to maintaining, upgrading, and monitoring by IT expert personals. In addition in the healthcare system, the cost of the software and hardware, inflexibility, complexity issues have raised the requirement for low cost technology to solve these issues (De la Torre-Díez et al., 2012).

Cloud computing is very useful for healthcare sector for reducing the complexity and facilitating the collaboration among the information systems in the healthcare sector. Cloud based architecture have capability to gathering the data from various sources, integrating, and analyzing the data in the real time and allows doctors to access to patient records in anyplace and anytime. Cloud computing is based on paying per use model and also using the cloud computing lead to reducing the startup cost of the hardware and software, networking, personals, and licensing purchasing. Cloud computing also offers the capability such as the disaster recovery, and backup data redundancy that are important and considerable for healthcare sector because it replicates the data in several locations for more robustness and availability (Chen et al., 2012; Al Masud \& Rashid, 2012; Mell \& Grance, 2009).

\subsection{SWOT Analysis for Cloud Computing with View to Healthcare Sector}

SWOT analysis is a strategic planning method to evaluate the strength, weakness, opportunities and threats that involved in the project. According to the objective of the project, identify the external and internal factors that are favorable and unfavorable to reach the objective. The key role of SWOT is helping a full awareness of all factors that may effect on strategic planning and decision making a goal that can be applied to most any aspect of industry. Nowadays, healthcare sector have plan to move to the cloud computing environment, so before moving the healthcare sector require to have some idea about the strengths, weakness, opportunities, and threats of cloud computing services. The SWOT analysis can provide some evidences (Guo et al., 2010). Table 1 shows the results of SWOT analysis for cloud computing in the healthcare sector.

\subsubsection{Strengths}

The Strengths of Cloud computing in the healthcare sector include: Cost effectiveness, Innovative and flexible, Simplify cost and consumption model, Compliant facilities, Flexible and resilient in disaster recovery, Maintenance cost reduction, Convenient level accessibility, Better control of resource, Time and location independence, Energy saving, Protection of environment, Friendly usage, and Ability to expand. While two of strengths are main and important that as below:

Using the cloud computing in the healthcare lead to cost reduction because does not need to start up expenditure. Healthcare faces with lack of the enough resources and also cannot affordable the intensive expenses. Cloud computing has capability to provide all of them.

Easy access to the patient's records in anytime and anywhere leads to increase collaboration among doctors and patients, so the quality of services to patients will be increased (Bamiah et al., 2012).

\subsubsection{Weaknesses}

The absence of the certain strengths may be viewed as weaknesses, so the weaknesses of the cloud computing in the healthcare sector contain: Training requirement, High speed internet connection requirement, Integrated with local software is very hard, Lack of the physical controlling of data, Lack of the commitment to control quality of service and availability, Application development and Increased dependence. While the main weakness of cloud computing in the healthcare sector as below:

Implementing the cloud computing in the healthcare sector is not easy task because they are many factors involved with adopting and implementing and also more depended to the cloud service provider. Many hospitals do not have even Internet connection to connect to the cloud, so in this case, it's very hard to implementing the cloud computing. Internet connection is fundamental and essential requirement for connecting to the cloud and use cloud service so without Internet the implementation the cloud computing for health sector is impossible (Rolim et al., 2010).

\subsubsection{Opportunities}

The Opportunities of the cloud computing in the healthcare sector include: User can use latest technology, Offer modern service for user, Modern and quick solution for problems, Good for healthcare sector because managing the progress with upfront investment, Standardize process, Adaptive to future requirements.

Two main and important opportunities of using the cloud computing in the healthcare sector as below: 
With using the cloud computing in the healthcare sector, healthcare sector personals can get opportunities to learn new technology.

Cloud computing is third party, if occur any problems, the cloud provider expert will provide quick solution without interruption hospital service (AbuKhousa et al., 2012).

\subsubsection{Threats}

Threats of cloud computing in the healthcare sector include: Security concerns (Data security), Loss of connectivity, Integration to another platform is hard, Hidden cost that include: Backup, Recover, and Problem solution, Lack of the specific standard regulation (local, national, and international), Reduction of compatibility Quality and performance of the healthcare personals are big concerns.

The main threats in adopting the cloud computing in the health sector is security concerns that related to the data security because the patient data that keep and process in the cloud is very sensitive and valuable so the security in the cloud is very important and its main barrier to adopt cloud computing in the healthcare sector (AbuKhousa et al., 2012; Deng et al., 2011; Zissis \& Lekkas, 2012).

\section{Conclusion}

Table 1. SWOT analysis for cloud computing in the healthcare sector. (AbuKhousa et al., 2012; Bamiah et al., 2012; Chowdhary et al., 2011;Deng et al., 2011; Haynie, 2009; Ratnam and Dominic, 2012; Rolim et al., 2010; Zissis \& Lekkas, 2012)

\begin{tabular}{l}
\hline Internal \\
\hline Strength \\
1) Cost effectiveness \\
2) Innovative and flexible \\
3) Simplify cost and consumption model \\
4) Compliant facilities \\
5) Flexible and resilient in disaster recovery \\
6) Maintenance cost reduction \\
7) Convenient level accessibility \\
8) Better control of resource \\
9) Time and location independence \\
10) Energy saving \\
11) Protection of environment \\
12) Friendly usage \\
13) Ability to expand
\end{tabular}

\section{Opportunities}

1) User can use latest technology

2) Offer modern service for user

3) Modern and quick solution for problems

4) Good for healthcare sector because managing the progress with upfront investment

\section{Weakness}

1) Training requirement

2) High speed internet connection requirement

3) Integrated with local software and software is very hard

4) Lack of the physical controlling of data

5) Lack of the commitment to control quality of service and availability
6) Application development
7) Increased dependence

Negative

\section{Threats}
1) Security concerns (Data security)
2) Loss of connectivity
3) Integration to another platform is hard
4) Hidden cost that include: backup, recover, and problem solution
5) Lack of the specific standard regulation (local, national, and international)
6) Reduction of compatibility
7) Quality and performance of the healthcare personals are big concerns

5) Standardize process

6) Adaptive to future requirements

\section{External}

From healthcare sector perspective the concept of cloud computing is not much accept because they are several issues involved, so in this paper, explain cloud computing conception, feature of it and healthcare need to using 
the cloud computing, and investigated on advantages and disadvantages of cloud computing. To achieve this, SWOT analysis was done in that strengths, weakness, opportunities, and threats of cloud computing in the healthcare sector was evaluated. The results of SWOT analysis presented that healthcare sector can make progress dramatically with cost reduction model and increase the quality of services. Lower investment on the infrastructure easy and fast access, optimizing the recourses are benefits of cloud computing solution for healthcare sector. Nevertheless, adopting the cloud computing is not free of risks, the major risk of cloud computing is lack of the data security and other important risk that some cloud user mention it, is a place of the data (data location). So, developing the strategies to provide more security can directly influence on enhancing the cloud computing adopting rate.

\section{Acknowledgments}

Research Management Center (RMC), universiti teknologi Malaysia (UTM), funded this research project (Project Id: Q.K130000.2540.05H77).

\section{References}

AbuKhousa, E., Mohamed, N., \& Al-Jaroodi, J. (2012). E-Health cloud: Opportunities and challenges. Future Internet, 4(3), 621-645. http://dx.doi.org/10.3390/fi4030621

Ahuja, S. P., \& Rolli, A. C. (2011). Survey of the State-of-the-Art of Cloud Computing. International Journal of Cloud Applications and Computing (IJCAC), 1(4), 34-43. http://dx.doi.org/10.4018/ijcac.2011100103

Al Masud, \& Rashid, S. M. (2012, May-June). A Novel Approach to Introduce Cloud Services in Healthcare Sectors for the Medically Underserved Populations in South Asia. International Journal of Engineering Research and Applications, 2(3), 1337-1346.

Bamiah, M., Brohi, S., Chuprat, S., \& AbManan, J. L. (2012, December). A study on significance of adopting cloud computing paradigm in healthcare sector. In Cloud Computing Technologies, Applications and Management (ICCCTAM), 2012 International Conference on (pp. 65-68). IEEE.

Chen, T. S., Liu, C. H., Chen, T. L., Chen, C. S., Bau, J. G., \& Lin, T. C. (2012). Secure Dynamic access control scheme of PHR in cloud computing. Journal of medical systems, 36(6), 4005-4020. http://dx.doi.org/10. 1007/s 10916-012-9873-8

Chowdhary, S. K., Yadav, A., \& Garg, N. (2011, April). Cloud computing: Future prospect for e-health. In Electronics Computer Technology (ICECT), 2011 3rd International Conference on (Vol. 3, pp. 297-299). IEEE.

De la Torre-Díez, I., Díaz-Pernas, F. J., Fernández, G., Antón-Rodríguez, M., Martínez-Zarzuela, M., González-Ortega, D., \& Boto-Giralda, D. (2012, May). Analysis of the benefits and constraints for the implementation of Cloud Computing over a EHRs system. In Proceedings of the 6th Euro American Conference on Telematics and Information Systems (pp. 151-156). ACM. http://dx.doi.org/10.1145/ 2261605.2261628

Deng, M., Petkovic, M., Nalin, M., \& Baroni, I. (2011, July). A Home Healthcare System in the Cloud--Addressing Security and Privacy Challenges.Cloud Computing (CLOUD), 2011 IEEE International Conference on (pp. 549-556). IEEE.

Fern'ndez, G., de la Torre-Díez, I., \& Rodrigues, J. J. (2012, July). Analysis of the Cloud Computing Paradigm on Mobile Health Records Systems. In Innovative Mobile and Internet Services in Ubiquitous Computing (IMIS), 2012 Sixth International Conference on (pp. 927-932). IEEE.

Guo, L., Chen, F., Chen, L., \& Tang, X. (2010, April). The building of cloud computing environment for e-health. In E-Health Networking, Digital Ecosystems and Technologies (EDT), 2010 International Conference on (Vol. 1, pp. 89-92). IEEE.

Haynie, M. (2009). Enterprise cloud services: Deriving business value from Cloud Computing. Micro Focus, Tech. Rep.

Koufi, V., Malamateniou, F., \& Vassilacopoulos, G. (2010, November). Ubiquitous access to cloud emergency medical services. In Information Technology and Applications in Biomedicine (ITAB), 2010 10th IEEE International Conference on (pp. 1-4). IEEE.

Mell, P., \& Grance, T. (2009). The NIST definition of cloud computing. National Institute of Standards and Technology, 53(6), 50.

Moschella, D., Neal, D., Opperman, P., \& Taylor, J. (2004, June). The "Consumerization" of Information 
Technology. Leading Edge Forum.

Ratnam, K. A., \& Dominic, P. D. D. (2012, June). Cloud services-Enhancing the Malaysian healthcare sector. In Computer \& Information Science (ICCIS), 2012 International Conference on (Vol. 2, pp. 604-608). IEEE.

Rolim, C. O., Koch, F. L., Westphall, C. B., Werner, J., Fracalossi, A., \& Salvador, G. S. (2010, February). A cloud computing solution for patient's data collection in healthcare institutions. In e-Health, Telemedicine, and Social Medicine, 2010.ETELEMED'10. Second International Conference on (pp. 95-99). IEEE.

Zissis, D., \& Lekkas, D. (2012). Addressing cloud computing security issues. Future Generation Computer Systems, 28(3), 583-592. http://dx.doi.org/10.1016/j.future.2010.12.006

\section{Copyrights}

Copyright for this article is retained by the author(s), with first publication rights granted to the journal.

This is an open-access article distributed under the terms and conditions of the Creative Commons Attribution license (http://creativecommons.org/licenses/by/3.0/). 